\title{
New lager yeast strains generated by interspecific hybridization
}

\author{
Kristoffer Krogerus • Frederico Magalhães • \\ Virve Vidgren • Brian Gibson
}

Received: 5 December 2014 / Accepted: 30 January 2015 / Published online: 15 February 2015

(C) The Author(s) 2015. This article is published with open access at Springerlink.com

\begin{abstract}
The interspecific hybrid Saccharomyces pastorianus is the most commonly used yeast in brewery fermentations worldwide. Here, we generated de novo lager yeast hybrids by mating a domesticated and strongly flocculent Saccharomyces cerevisiae ale strain with the Saccharomyces eubayanus type strain. The hybrids were characterized with respect to the parent strains in a wort fermentation performed at temperatures typical for lager brewing $\left(12{ }^{\circ} \mathrm{C}\right)$. The resulting beers were analysed for sugar and aroma compounds, while the yeasts were tested for their flocculation ability and $\alpha$-glucoside transport capability. These hybrids inherited beneficial properties from both parent strains (cryotolerance, maltotriose utilization and strong flocculation) and showed apparent hybrid vigour, fermenting faster and producing beer with higher alcohol content (5.6 vs $4.5 \% \mathrm{ABV}$ ) than the parents. Results suggest that interspecific hybridization is suitable for production of novel non-GM lager yeast strains with unique properties and will help in elucidating the evolutionary history of industrial lager yeast.
\end{abstract}

Keywords Lager yeast $\cdot$ S. eubayanus $\cdot$ Brewing · Hybrid $\cdot$ Mating $\cdot$ Heterosis

Electronic supplementary material The online version of this article (doi:10.1007/s10295-015-1597-6) contains supplementary material, which is available to authorized users.

K. Krogerus $(\bowtie) \cdot$ F. Magalhães $\cdot$ V. Vidgren $\cdot$ B. Gibson VTT Technical Research Centre of Finland, Tietotie 2, P.O. Box 1000, 02044 Espoo, Finland e-mail: kristoffer.krogerus@aalto.fi

\section{K. Krogerus · F. Magalhães}

Department of Biotechnology and Chemical Technology, School of Chemical Technology, Aalto University, Kemistintie 1, Aalto, P.O. Box 16100, 00076 Espoo, Finland

\section{Introduction}

Pale lager is the most popular beer style worldwide. All beers of this type are produced through the fermentation of malt wort at low temperature with the interspecific hybrid yeast species Saccharomyces pastorianus [14]. The formation of interspecific hybrids is common among the closely related species of the Saccharomyces genus, and the lager yeast $S$. pastorianus is a natural hybrid between $S$. cerevisiae and the newly discovered S. eubayanus [21]. Hybrid species are common in anthropogenic environments and are not limited to yeast, as hybrid species often exhibit qualities superior to both parent strains [22,33]. Lager beers have gained popularity due to their 'clean' flavour profile, i.e. lack of ester-derived fruity/floral aroma, resulting mainly from fermentation at low temperatures. Ale strains (S. cerevisiae) have over time been selected for beneficial brewing properties, such as flocculation, sugar utilization, and flavour profile, but require warmer temperatures $\left(>15^{\circ} \mathrm{C}\right)$ for optimal fermentation [40]. In contrast, S. eubayanus thrives at the low temperatures responsible for the typical aroma profile of lager beer, but lacks the ability to utilize maltotriose (the most abundant sugar in wort after maltose) and exhibits poor flocculation [13].

The origins of lager yeast are not known, but it may be possible that the original hybridization event was due to $S$. eubayanus contaminating a traditional ale fermentation with $S$. cerevisiae [14]. The $S$. pastorianus group consists of at least two distinct lineages (Saaz and Frohberg), which may have arisen independently $[11,23]$ or through a common hybridization event [42]. While these two groups share many common features, they differ functionally in a number of respects, such as maltotriose utilization and cold tolerance $[13,34]$. These functional differences seem to be reflected in the genomic difference between 
the groups (Saaz strains have retained proportionally more DNA derived from the S. eubayanus parent, while Frohberg strains have retained proportionally more DNA from the $S$. cerevisiae parent). Because lager yeasts are restricted to only these two genetically distinct groups, the genetic diversity among them is poor. However, through the selection of individual $S$. eubayanus and $S$. cerevisiae strains with specific desirable characteristics, and subsequent hybridization of these strains, it may be possible to produce novel, tailor-made yeast strains immediately available for industrial use.

The use of interspecific hybridization for generating ale and wine yeast strains with improved aroma production has been described $[1,12,38,39]$. We here describe, for the first time, the use of mass mating to generate de novo lager yeast hybrids from a domesticated strongly flocculent $S$. cerevisiae ale strain and the S. eubayanus type strain. The hybrids were characterized with respect to the parent strains in a wort fermentation performed at temperatures typical for lager yeast, and the resulting beer was analysed for sugar content and the yeast was tested for its flocculation ability. The aim of this study was to demonstrate the generation of novel lager yeast strains with physiological properties inherited from both parental strains. This technique has the potential to greatly increase the diversity of yeast strains available for lager brewing [14]. The yeast hybrids generated with this technique can also be seen as non-GM, and their use is thus not prevented by legislation or public opinion [6].

\section{Materials and methods}

\section{Yeast strains}

The two parental strains were $S$. cerevisiae VTT-A81062 (VTT Culture Collection, Finland), a brewer's yeast strain originally sourced from an ale beer from the United Kingdom, and the $S$. eubayanus type strain VTT-C12902 (VTT Culture Collection, Finland; deposited as CBS12357 at CBS-KNAW Fungal Biodiversity Centre). The four hybrid strains $(\mathrm{A} 81062 \times \mathrm{C} 12902)$ that were chosen for further characterization were named $\mathrm{H} 1-\mathrm{H} 4$. Natural auxotrophic mutants (lys- and ura-) of the parental strains were selected on $\alpha$-aminoadipic and 5-fluoroorotic acid agar plates, respectively $[4,50]$. Auxotrophy was confirmed by the inability to grow on minimal selection agar medium $(0.67 \%$ Yeast Nitrogen Base without amino acids, $3 \%$ glycerol, $3 \%$ ethanol and $2 \%$ agar).

\section{Sporulation}

For the generation of ascospores, the auxotrophic mutants of the parental strains were first grown overnight in YPM medium ( $1 \%$ yeast extract, $2 \%$ peptone, $4 \%$ maltose) at $20{ }^{\circ} \mathrm{C}$. The yeast was then inoculated into pre-sporulation medium ( $0.8 \%$ yeast extract, $0.3 \%$ peptone, $10 \%$ glucose $)$ at an OD600 of 0.3 and allowed to grow for $20 \mathrm{~h}$ at $20^{\circ} \mathrm{C}$. The yeast was then washed with $1 \%$ potassium acetate and a thick suspension was plated onto sporulation agar $(1 \%$ potassium acetate, $10 \mathrm{mg} / \mathrm{L}$ lysine and uracil, $2 \%$ agar). The yeast was allowed to sporulate for 7 days at $25{ }^{\circ} \mathrm{C}$. Sporulation efficiency was calculated by counting the frequency of ascospores stained with malachite green [27]. Spore viability was calculated by dissecting ascospores treated with Zymolyase 100T (US Biological, USA) on YPD agar with a micromanipulator [43].

\section{Generation of interspecific hybrids}

Interspecific hybrids between a ura- isolate of $S$. cerevisiae A81062 and a lys- isolate of S. eubayanus C12902 were produced by first generating ascospores of the auxotrophic mutants as above. Ascospores were scraped off the agar into $1 \mathrm{ml}$ sterile reverse-osmosis purified $\mathrm{H}_{2} \mathrm{O}$ in $2 \mathrm{ml}$ Eppendorf tubes. Tubes were centrifuged at $5,000 \times g$ for $5 \mathrm{~min}$ and the supernatant was removed. Ascus walls were digested by the addition of $50 \mu 11 \mathrm{mg} / \mathrm{ml}$ Zymolyase $100 \mathrm{~T}$ and incubation at $30{ }^{\circ} \mathrm{C}$ for $20 \mathrm{~min} .200 \mu \mathrm{l}$ of sterile $\mathrm{H}_{2} \mathrm{O}$ was then added, and cells were resuspended by vortexing. $100 \mu \mathrm{l}$ of the resulting suspensions from both parental strains, with complementary auxotrophic markers, were transferred together to $1 \mathrm{ml} \mathrm{YPM}$ medium in a sterile $2 \mathrm{ml}$ Eppendorf tube. Tubes were vortexed and incubated statically at $25{ }^{\circ} \mathrm{C}$ for 7 days. After incubation, the tubes were centrifuged at $5,000 \times g$ for $5 \mathrm{~min}$ and the supernatant was removed. $500 \mu \mathrm{l}$ of starvation medium $(0.1 \%$ yeast extract and $0.1 \%$ glucose) was added, and tubes were incubated for at least $2 \mathrm{~h}$ at room temperature. Tubes were then vortexed, after which the approximate cell concentration of the resulting suspension was measured with a NucleoCounter YC-100 (ChemoMetec, Denmark) and $100 \mu \mathrm{l}$ aliquots were spread onto minimal selection agar (without uracil or lysine). Plates were incubated at $25{ }^{\circ} \mathrm{C}$, and prototrophic colonies (i.e. potential hybrids) appeared after 3-7 days. Colonies were counted and purified by replating on minimal selection agar.

\section{Confirmation of hybrid status by PCR and RFLP}

The hybrid status of isolates was confirmed by amplification of rDNA-PCR (ITS1, 5.8S and ITS2) using the primers ITS1 ( $5^{\prime}$-TCCGTAGGTGAACCTGCGG- $\left.3^{\prime}\right)$ and ITS4 $\left(5^{\prime}\right.$-TCCTCCGCTTATTGATATGC- $\left.3^{\prime}\right)$ and digestion of amplicons using the HaeIII restriction enzyme (New England BioLabs, USA) as described previously [31]. Identification was based on the number of restriction fragments 
generated by enzyme digestion. S. eubayanus yielded a 3-band pattern (490, 225, 140 bp), S. cerevisiae a 4-band pattern $(320,225,180,140 \mathrm{bp})$, while successful hybrids yielded a pattern with all 5 bands (490, 320, 225, 180 and $140 \mathrm{bp})$.

Amplification of the S. eubayanus-specific FSYI gene (amplicon size $228 \mathrm{bp}$ ) and the $S$. cerevisiaespecific MEX67 gene (amplicon size 150 bp) was also performed on the DNA extracted from the hybrid strains using the primers SeubF3 (5'-GTCCCTGTAC CAATTTAATATTGCGC- $3^{\prime}$ ), SeubR2 (5'-TTTCACATCT CTTAGTCTTTTCCAGACG-3'), ScerF2 (5'-GCGCTTTA CATTCAGATCCCGAG- $3^{\prime}$ ), and ScerR2 (5'-TAAGTTG GTTGTCAGCAAGATTG- $3^{\prime}$ ) as described by Muir et al. [28] and Pengelly and Wheals [30]. Hybrids were i€dentified by the presence of both genes.

\section{Confirmation of hybrid status by PFGE}

Yeast strains were propagated in YPM at $20{ }^{\circ} \mathrm{C}$ to an OD600 $>1$ and then harvested by centrifugation $(3,000 \times g$, 5 min, $4{ }^{\circ} \mathrm{C}$ ). Supernatants were decanted, and cells were resuspended in $10 \mathrm{ml}$ of $4{ }^{\circ} \mathrm{C} 50 \mathrm{mM}$ EDTA (pH 8). Cell concentrations were determined with a Nucleocounter $^{\circledR}$ YC-100 ${ }^{\mathrm{TM}}$ (ChemoMetec) and $1.2 \times 10^{8}$ cells were placed in each sample plug. Sample plugs were prepared with the CHEF Genomic DNA Plug Kit for Yeast (BioRad) according to the manufacturer's instructions.

Sample plugs were loaded into the wells of a $1.0 \%$ pulse field certified agarose (Bio-Rad) gel. PFGE was performed at $14{ }^{\circ} \mathrm{C}$ in $0.5 \times$ TBE buffer [ $89 \mathrm{mMTris}, 89 \mathrm{mM}$ boric acid, 2 mM EDTA (pH 8)]. A CHEF Mapper XA pulsed field electrophoresis system (Bio-Rad) was used with the following settings: $6 \mathrm{~V} / \mathrm{cm}$ in a $120^{\circ}$ angle, pulse length increasing linearly from 26 to $228 \mathrm{~s}$, and total running time of $38 \mathrm{~h}$. A commercial chromosome marker preparation from S. cerevisiae strain YNN295 (Bio-Rad) was used for molecular mass calibration. After electrophoresis, the gels were stained with ethidium bromide and scanned with Gel Doc XR+ imaging system (Bio-Rad).

DNA content by flow cytometry

Flow cytometry was performed on the yeast strains essentially as described by Haase and Reed [16]. Cells were grown overnight in YPD medium $(1 \%$ yeast extract, $2 \%$ peptone, $2 \%$ glucose), and approximately $1 \times 10^{7}$ cells were washed with $1 \mathrm{~mL}$ of $50 \mathrm{mM}$ citrate buffer. Cells were then fixed with cold $70 \%$ ethanol and incubated at room temperature for $1 \mathrm{~h}$. Cells were then washed with $50 \mathrm{mM}$ citrate buffer, resuspended in $50 \mathrm{mM}$ citrate buffer containing $0.25 \mathrm{mg} \mathrm{mL}^{-1}$ RNAse A and incubated overnight at $37{ }^{\circ} \mathrm{C} .1 \mathrm{mg} \mathrm{mL}{ }^{-1}$ of Proteinase $\mathrm{K}$ was then added, and cells were incubated for $1 \mathrm{~h}$ at $50{ }^{\circ} \mathrm{C}$. Cells were then stained with SYTOX Green $(2 \mu \mathrm{M}$; Life Technologies, USA), and their DNA content was determined using a FACSAria cytometer (Becton-Dickinson). DNA contents were estimated by comparing fluorescence intensities with those of S. cerevisiae haploid (CEN.PK113-1A) and diploid (CEN.PK) reference strains. Measurements were performed on duplicate independent yeast cultures, and 100,000 events were collected per sample during flow cytometry.

\section{Characterization of hybrid strains}

Four randomly selected hybrids (H1-H4) and the parental strains were chosen for further characterization in a smallscale wort fermentation performed at $12{ }^{\circ} \mathrm{C}$. Yeast was propagated essentially as described previously [19], with the use of a 'Generation 0' fermentation prior to the actual experimental fermentations. The experimental fermentations were carried out in duplicate, in 2-L cylindroconical stainless steel fermenting vessels, containing $1.5 \mathrm{~L}$ of wort medium. The wort was produced at the VTT Pilot Brewery from barley malt and wheat malt, and contained an extract content of $12.0^{\circ}$ Plato $(59 \mathrm{~g}$ maltose, $19 \mathrm{~g}$ maltotriose, $16 \mathrm{~g}$ glucose, and $4.6 \mathrm{~g}$ fructose per litre) and free amino nitrogen (FAN) content of $269 \mathrm{mg} \mathrm{L}^{-1}$. Yeast was inoculated at a rate of $4 \mathrm{~g}$ fresh yeast per litre of wort (corresponding to $16 \times 10^{6}$ viable cells $\mathrm{mL}^{-1}$ ). The wort was oxygenated to $18 \mathrm{mg} \mathrm{L}^{-1}$ prior to pitching. The fermentations were carried out at $12{ }^{\circ} \mathrm{C}$ for 11 days, or until no change in residual extract was observed for $24 \mathrm{~h}$. Wort samples were drawn regularly from the fermentation vessels with a syringe, and placed directly on ice, after which the yeast was separated from the fermenting wort by centrifugation $(9,000 \times g$, $10 \min , 1^{\circ} \mathrm{C}$ ).

Flocculation of the yeast strains was evaluated using a modified Helm's assay essentially as described by D'Hautcourt and Smart [9]. Cultures recovered from fermentation were washed twice with 0.5 M EDTA ( $\mathrm{pH} 7$ ) to break the cell aggregates and then diluted to an OD600 of 0.4 . Flocculation was assayed by first washing yeast pellets with $4 \mathrm{mM} \mathrm{CaCl}_{2}$ solution and resuspending them in $1 \mathrm{ml}$ of flocculation solution containing $4 \mathrm{mM} \mathrm{CaCl}_{2}, 6.8 \mathrm{~g} / \mathrm{L}$ sodium acetate, $4.05 \mathrm{~g} / \mathrm{L}$ acetic acid, and $4 \%(\mathrm{v} / \mathrm{v})$ ethanol ( $\mathrm{pH} 4.5)$. Yeast cells in control tubes were resuspended in $0.5 \mathrm{M}$ EDTA ( $\mathrm{pH} 7$ ). After a sedimentation period of $10 \mathrm{~min}$, samples $(200 \mu \mathrm{L})$ were taken from just below the meniscus and dispersed in $10 \mathrm{mM}$ EDTA $(800 \mu \mathrm{L})$. The absorbance at $600 \mathrm{~nm}$ was measured, and percentage of flocculation was determined from the difference in absorbance between control and flocculation tubes.

Maltose and maltotriose uptake by the yeast strains was assayed by first growing them in YPM medium at $20{ }^{\circ} \mathrm{C}$. Yeasts were usually harvested at an OD600 $\mathrm{nm}$ between 4 
and 8 (i.e. at $2 \pm 1 \mathrm{mg}$ dry yeast $\mathrm{mL}^{-1}$ ) by centrifugation, washed with ice-cold water and then with ice-cold $0.1 \mathrm{M}$ tartrate-Tris ( $\mathrm{pH} 4.2$ ) and finally resuspended in the same buffer at a concentration of $200 \mathrm{mg}$ of fresh yeast $\mathrm{mL}^{-1}$. Zero-trans rates of $\left[\mathrm{U}_{-}{ }^{14} \mathrm{C}\right]$-maltose and $\left[\mathrm{U}_{-}{ }^{14} \mathrm{C}\right]$-maltotriose uptake at $20{ }^{\circ} \mathrm{C}$ were determined with $5 \mathrm{mM}$ substrate in $0.1 \mathrm{M}$ tartrateTris ( $\mathrm{pH} \mathrm{4.2)} \mathrm{as} \mathrm{described} \mathrm{earlier} \mathrm{[24],} \mathrm{with} \mathrm{reaction} \mathrm{time} \mathrm{of}$ 1 min. $\left[\mathrm{U}_{-}{ }^{14} \mathrm{C}\right]$-maltose (ARC 488 ) and $\left[\mathrm{U}_{-}{ }^{14} \mathrm{C}\right]$-maltotriose (ARC 627) were from American Radiolabeled Chemicals Inc. (St. Louis, MO, USA). $\left[\mathrm{U}-{ }^{14} \mathrm{C}\right]-$ maltotriose was repurified before use as described by Dietvorst et al. [10].

\section{Wort and beer analysis}

The specific gravity, alcohol level and $\mathrm{pH}$ of samples were determined from the centrifuged and degassed fermentation samples using an Anton Paar density meter DMA $5000 \mathrm{M}$ (Anton Paar GmbH, Austria) with Alcolyzer Beer ME and pH ME modules (Anton Paar GmbH, Austria).

The yeast dry mass content of the samples (i.e. yeast in suspension) was determined by washing the yeast pellets gained from centrifugation twice with $25 \mathrm{~mL}$ deionized $\mathrm{H}_{2} \mathrm{O}$ and then suspending the washed yeast in a total of $6 \mathrm{~mL}$ deionized $\mathrm{H}_{2} \mathrm{O}$. The suspension was then transferred to a pre-weighed porcelain crucible and was dried overnight at $105^{\circ} \mathrm{C}$ and allowed to cool in a desiccator, before the change of mass was measured.

Concentrations of fermentable sugars (glucose, fructose, maltose and maltotriose) were measured by HPLC using a waters 2695 separation module and waters system interphase module liquid chromatograph coupled with a waters 2414 differential refractometer (Waters Co., Milford, MA, USA). An Aminex HPX-87H organic acid analysis column $(300 \times 7.8 \mathrm{~mm}$, Bio-Rad $)$ was equilibrated with $5 \mathrm{mM} \mathrm{H}_{2} \mathrm{SO}_{4}$ (Titrisol, Merck, Germany) in water at $55^{\circ} \mathrm{C}$ and samples were eluted with $5 \mathrm{mM} \mathrm{H} \mathrm{H}_{2} \mathrm{SO}_{4}$ in water at a $0.3 \mathrm{ml} / \mathrm{min}$ flow rate.

Yeast-derived flavour compounds were determined by headspace gas chromatography with flame ionization detector (HS-GC-FID) analysis. $4 \mathrm{~mL}$ of samples was filtered $(0.45 \mu \mathrm{m})$, incubated at $60{ }^{\circ} \mathrm{C}$ for $30 \mathrm{~min}$ and then $1 \mathrm{~mL}$ of gas phase was injected (split mode; $225^{\circ} \mathrm{C}$; split flow of $30 \mathrm{~mL} \mathrm{~min}^{-1}$ ) into a gas chromatograph equipped with a FID detector and headspace autosampler (Agilent 7890 Series; Palo Alto, CA, USA). Analytes were separated on a HP-5 capillary column $(50 \mathrm{~m} \times 320 \mu \mathrm{m} \times 1.05 \mu \mathrm{m}$ column, Agilent, USA). The carrier gas was helium (constant flow of $1.4 \mathrm{~mL} \mathrm{~min}^{-1}$ ). The temperature program used $50{ }^{\circ} \mathrm{C}$ for $3 \mathrm{~min}, 10^{\circ} \mathrm{C} \mathrm{min}{ }^{-1}$ to $100{ }^{\circ} \mathrm{C}, 5{ }^{\circ} \mathrm{C} \mathrm{min}{ }^{-1}$ to $140{ }^{\circ} \mathrm{C}, 15^{\circ} \mathrm{C} \mathrm{min}{ }^{-1}$ to $260{ }^{\circ} \mathrm{C}$ and then isothermal for $1 \mathrm{~min}$. Compounds were identified by comparison with authentic standards and were quantified using standard curves. 1-Butanol was used as internal standard.

\section{Results and discussion}

Generation of interspecific hybrids

Auxotrophic mutants of the parental strains were successfully generated and the mutant strains, A81062 ura- and C12902 lys-, produced ascospores on the solid sporulation medium. However, quite low sporulation efficiencies were observed for the A81062 ura- strain compared to the C12902 lys- strain (11 and $78 \%$, respectively). These ascospores did contain viable spores though (12 and $71 \%$, respectively) and were used to generate the interspecific hybrids. After mating and spreading on selection plates, the first colonies started to emerge after 3 days. After a week, a total of 38 colonies (corresponding to a hybridization frequency of $2.6 \times 10^{-6}$ ) were obtained.

\section{Confirmation of hybrid status}

After amplification of the rDNA and digestion with HaeIII, a 4-band pattern was obtained for the $S$. cerevisiae A81062 parental strain, a 3-band pattern was obtained for the $S$. eubayanus C12902, while 5-band patterns were obtained for the isolated hybrids (Fig. 1a), which confirms that both parental genomes were represented in these hybrids. This RFLP profile of the hybrids is different to that of industrial S. pastorianus hybrids which, as a result of post-hybridization DNA loss, exhibit either the 3-band or 4-band pattern depending on whether they belong to the Saaz or Frohberg group [14, 31]. Amplification of FSYI and MEX67 genes

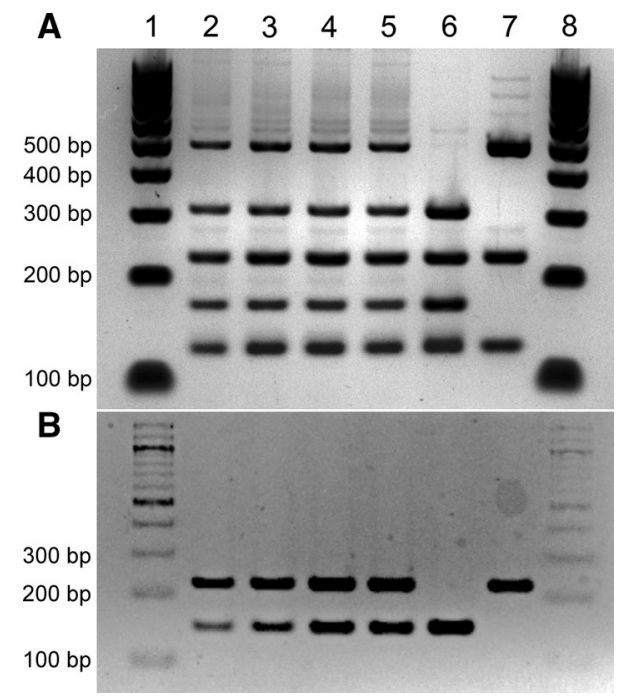

Fig. 1 Confirmation of hybridization by a rDNA ITS PCR and RFLP, and $\mathbf{b}$ amplification of FSY1 and MEX67 genes. Lanes 1 and 8, 100 bp DNA ladder, lane 2-5 hybrids $\mathrm{H} 1-\mathrm{H} 4$, lane $6 \mathrm{~S}$. cerevisiae A81062 parental strain, and lane 7 S. eubayanus C12902 parental strain 

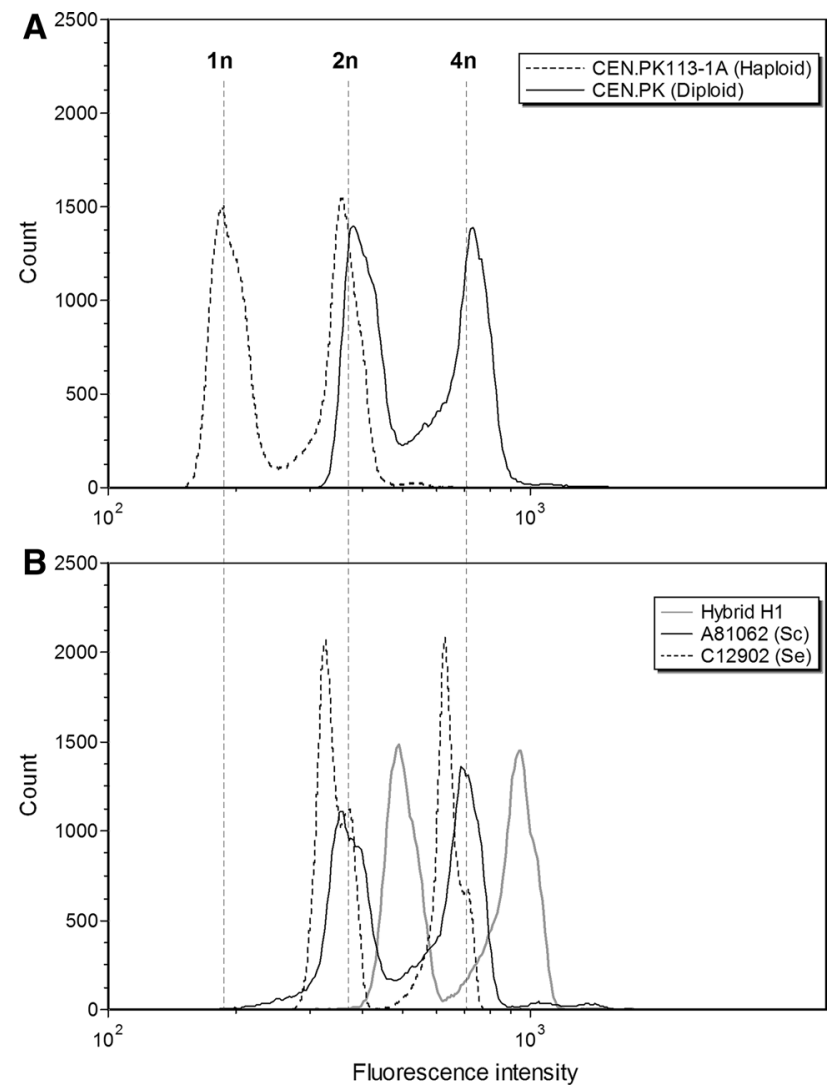

Fig. 2 DNA content of the a S. cerevisiae haploid (CEN.PK113-1A) and diploid (CEN.PK) reference strains, and b hybrid strain $\mathrm{H} 1$ and parent strains by flow cytometry. The other hybrid strains $\mathrm{H} 2-\mathrm{H} 4$ showed identical profiles to that of hybrid $\mathrm{H} 1$. The dashed vertical lines represent the approximate fluorescence intensity corresponding to $1 \mathrm{n}, 2 \mathrm{n}$ and $4 \mathrm{n}$ DNA content

by PCR (Fig. 1b), also revealed the presence of $S$. eubayanus and $S$. cerevisiae genes, respectively, in the genomes of the hybrid strains. Hybrid status was then finally confirmed with PFGE, which also suggested that the hybrid strains had inherited a complete set of chromosomes from both parent strains (shown in Supplementary material; Figure S1). Flow cytometry further revealed that all four hybrid strains were most likely triploid (Fig. 2). For clarity, only the fluorescence profile of hybrid strain $\mathrm{H} 1$ (of the hybrid strains) is shown in Fig. 2b, but the other hybrid strains $\mathrm{H} 2-\mathrm{H} 4$ produced identical profiles. This is in contrast to what would be expected from haploid sporeto-spore matings, which result in the formation of diploid cells. Here, hybridization was apparently a result of raremating between a haploid spore from one parent and a diploid cell, which may have undergone loss of heterozygosity and conversion to $a / a$ or $\alpha / \alpha$ mating type, from the other parent [15]. This also explains the relatively low hybridization frequency [1]. It is not known what portions of the parent genomes have been inherited in the hybrid strains, but it is likely that the four hybrid strains $\mathrm{H} 1-\mathrm{H} 4$ contained 2n DNA from the $S$. cerevisiae A81062 strain and 1n DNA from the S. eubayanus C12902 strain, because of the higher sporulation efficiency and spore viability of C12902. However, the data collected here are not enough to show this, and it is also possible that the hybrid strains contain proportionally more DNA from the $S$. eubayanus parent. The proportionality of DNA inheritance might be directly reflected in the functional properties of the hybrid strains, as seems to be the case for Saaz and Frohberg lager yeasts [13].

Fermentation characteristics of hybrid strains

All four hybrid strains (H1-H4) successfully fermented the wort at $12{ }^{\circ} \mathrm{C}$ and displayed fermentation rates higher than both parent strains (Fig. 3). Fermentations with hybrid strains $\mathrm{H} 1$ and $\mathrm{H} 2$ were complete after 8 days. Not only did the hybrid strains ferment faster, but they also produced beers with higher alcohol content (5.6 vs $4.5 \% \mathrm{ABV}$ after 11 days of fermentation). The $S$. cerevisiae A81062 parent strain grew and fermented slowly at $12{ }^{\circ} \mathrm{C}$ and hence only reached an $\mathrm{ABV}$ of $4.2 \%$ after 11 days. The successful growth and fermentation of the hybrid strains at $12{ }^{\circ} \mathrm{C}$ suggest that the cold tolerance of $S$. eubayanus has been transferred to the hybrid strains. The mechanisms that govern $S$. eubayanus cryotolerance are unknown, but it is most likely related to differences in the membrane composition [17] as well as product activity and expression of central metabolic genes [29], compared to other Saccharomyces yeast. Similar trends in fermentation profiles were observed during the 'Generation 0' fermentation performed at $20^{\circ} \mathrm{C}$, as the hybrid strains exhibited improved fermentation rates compared to both parent strains (data not shown). Furthermore, all four hybrid strains and the A81062 parent strain grew at $37{ }^{\circ} \mathrm{C}$, but not $40{ }^{\circ} \mathrm{C}$, on YPM plates, while $S$. eubayanus $\mathrm{C} 12902$ grew at neither temperature, suggesting the hybrid strains also inherited heat tolerance from the ale parent. The cause of the apparent heterotic phenotype of the hybrids could be differential functionality and expression of orthologous genes derived from the two parents (specifically linked to central carbon metabolism and sugar transport), as well as different temperature optima of the gene products $[7,14]$. The small difference in fermentation performance between the hybrid strains could be a result of meiotic segregation during spore formation or differential inheritance of mitochondrial DNA, as mtDNA is inherited from only one parent $[25,35]$.

The sugar profiles of the original wort and the beers (Table 1) reveal that the higher attenuation reached by the hybrid strains compared to the $S$. eubayanus parent is a result of differential maltotriose utilization. The ability to ferment maltotriose seems to have been inherited from the $S$. cerevisiae parent, as the active sugar transport 

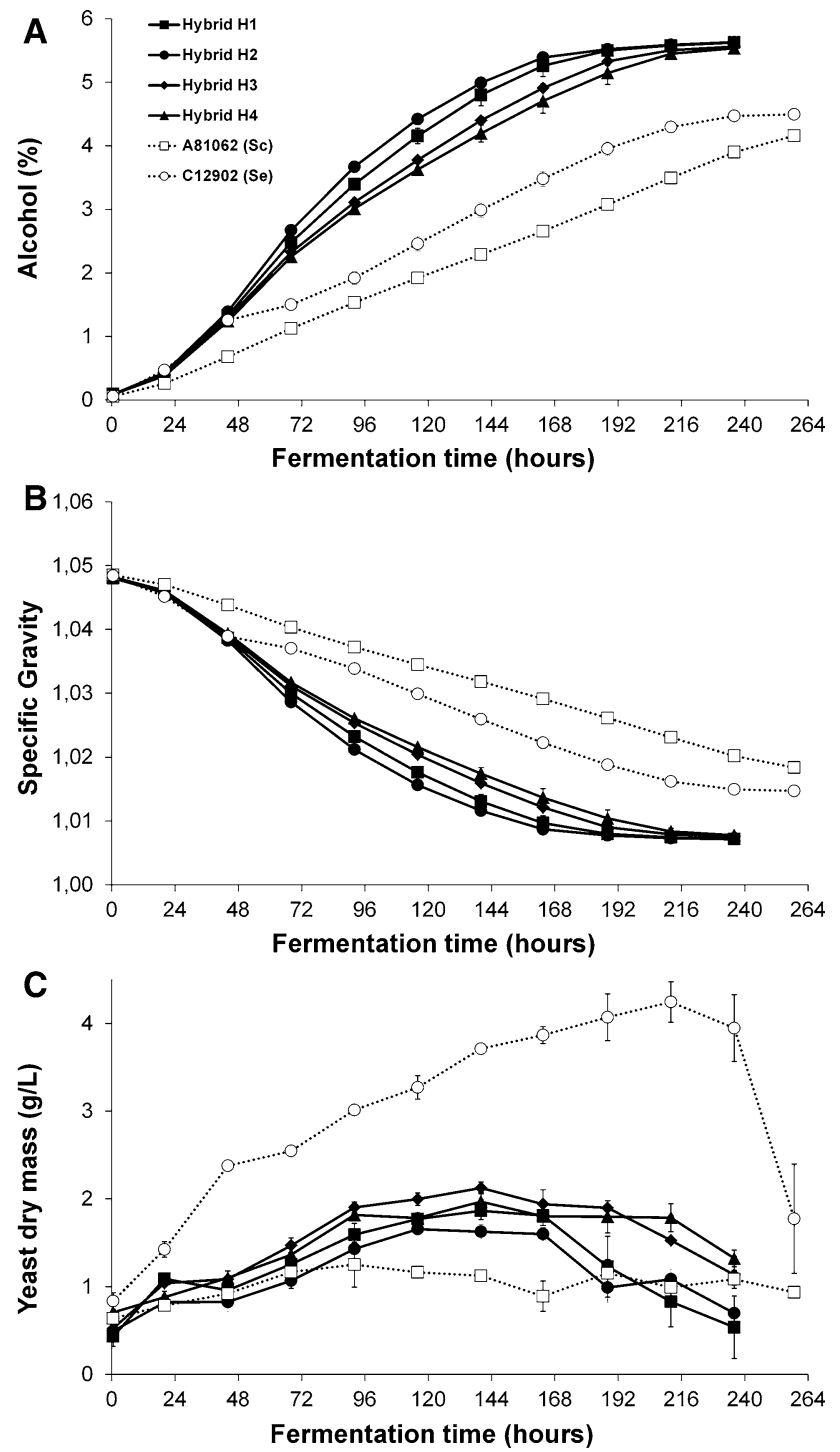

Fig. 3 a The alcohol content (\% ABV), b specific gravity and $\mathbf{c}$ suspended yeast dry mass $(\mathrm{g} / \mathrm{L})$ of the $12{ }^{\circ} \mathrm{P}$ wort fermented with the hybrid strains (solid lines) and parent strains (dotted lines). Values are means from two independent fermentations and error bars where visible represent the range

Table 1 Sugars $(\mathrm{g} / \mathrm{L})$ in the original wort and beers fermented with the hybrid and parent strains

\begin{tabular}{lrr}
\hline Yeast strain/sample & \multicolumn{1}{l}{ Maltose } & Maltotriose \\
\hline Original wort & $59.1( \pm 0.37)$ & $18.8( \pm 0.21)$ \\
Hybrid H1 & $1.6( \pm 0.04)$ & $3.6( \pm 0.07)$ \\
Hybrid H2 & $1.5( \pm 0.03)$ & $3.4( \pm 0.05)$ \\
Hybrid H3 & $1.6( \pm 0.02)$ & $3.9( \pm 0.13)$ \\
Hybrid H4 & $1.6( \pm 0.20)$ & $5.3( \pm 0.24)$ \\
A81062 & $25.1( \pm 0.69)$ & $5.5( \pm 0.09)$ \\
C12902 & $1.8( \pm 0.15)$ & $18.5( \pm 0.58)$ \\
\hline
\end{tabular}

Values are means from two independent fermentations (standard deviation in parenthesis) assays confirmed that all the hybrid strains and $S$. cerevisiae A81062 were able to transport this sugar across the cell membrane, while S. eubayanus $\mathrm{C} 12902$ showed negligible uptake (Table 2) as shown previously by Gibson et al. [13]. Maltotriose uptake is common in lager yeast strains; however, this ability seems to be limited to strains belonging to the Frohberg group [13]. Maltose and maltotriose use during fermentation are dependent on the activity of a range of transmembrane transporters [46]. In industrial ale strains maltotriose is essentially carried into the cell by $A G T 1$ transporters, while it has been found in lager yeast strains that $S$. cerevisiae-derived $A G T 1$ genes remain non-functional, and maltotriose transport is rather thought to be carried out by Mtt1 (Mty1) and a diverged form of $A G T 1$, which have presumably both been inherited from the $S$. eubayanus parent $[10,45,46,48]$. This contradicts the fact that negligible transport of maltotriose into the $S$. eubayanus type strain was observed here and in previous studies [13], but the maltotriose use of other S. eubayanus strains, e.g. from different geographical origins, currently remains unexplored. The utilization of maltotriose is of importance during brewery fermentations because it is the second most abundant sugar in wort, its utilization results in a higher alcohol yield and residual maltotriose can affect flavour [51]. The fermentation profiles (Fig. 3a, b) reveal that the hybrid strains and S. eubayanus exhibited a similar fermentation rate in the beginning of fermentation, when monosaccharides are predominately taken up and utilized by the yeast [3]. After the first $48 \mathrm{~h}$, the fermentation rates of the hybrid strains increase relative to $S$. eubayanus, presumably because of more efficient maltose and maltotriose utilization in the former. The hybrid strains and S. eubayanus exhibited fermentation rates greater than $S$. cerevisiae during essentially the entire fermentation because the temperature is clearly sub-optimal for S. cerevisiae. This is also evident from the fact that there was residual maltose in the beer fermented with $S$. cerevisiae (Table 1). Here, a relatively low-gravity wort ( $12^{\circ}$ Plato) was used, but industry is showing interest towards higher gravity worts [37]. While the fermentation performance of the hybrid strains in high-gravity wort was not tested in this study, it may be possible that they possess increased stress tolerance as a result of their polyploidy [52].

The concentrations of aroma compounds in the beers (Fig. 4) reveal that all four hybrid strains produced beers with similar aroma profiles. Compared to the hybrid strains, S. eubayanus C12902 produced more of the higher alcohols related to branched-chain amino acid synthesis (almost twofold concentrations of 2-methylpropanol, 3-methylbutanol and 2-methylbutanol). These give the beer alcoholic and solvent-like aromas, which are generally considered unpleasant [32]. Despite these twofold differences in 3-methylbutanol concentrations, the 
Table 2 The flocculation ability and maltose and maltotriose uptake at $20^{\circ} \mathrm{C}$ by the hybrid and parent strains

An uptake activity $\leq 0.5 \mu \mathrm{mol} \mathrm{min} \mathrm{m}^{-1} \mathrm{~g}^{-1} \mathrm{DY}$ is considered negligible. Values are means of three independent assays (standard deviation in parenthesis)

\begin{tabular}{|c|c|c|c|}
\hline Yeast strain & Flocculation ability (\%) & $\begin{array}{l}\text { Maltose uptake } \mu \mathrm{mol} \mathrm{min}^{-1} \mathrm{~g}^{-1} \\
\text { DY ( } 5 \mathrm{mM} \text { maltose) }\end{array}$ & 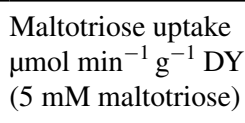 \\
\hline Hybrid H1 & $85( \pm 2.1)$ & $12.6( \pm 1.7)$ & $4.1( \pm 0.3)$ \\
\hline Hybrid H2 & $87( \pm 1.3)$ & $12.3( \pm 3.0)$ & $4.1( \pm 0.2)$ \\
\hline Hybrid H3 & $88( \pm 1.6)$ & $15.8( \pm 0.9)$ & $5.4( \pm 1.4)$ \\
\hline Hybrid H4 & $82( \pm 4.5)$ & $16.1( \pm 0.7)$ & $5.4( \pm 1.3)$ \\
\hline A81062 & $71( \pm 4.2)$ & $10.1( \pm 0.5)$ & $4.2( \pm 0.2)$ \\
\hline C12902 & $15( \pm 0.8)$ & $13.2( \pm 3.1)$ & $0.1( \pm 0.0)$ \\
\hline
\end{tabular}

concentrations of 3-methylbutyl acetate were quite similar for the hybrid strains and S. eubayanus (around the flavour threshold of $1.2 \mathrm{mg} / \mathrm{L}$ [26]). 3-Methylbutyl acetate gives beer a banana- and pear-like aroma, which is considered desirable in several beer styles [32]. The hybrid strains produced more ethyl esters than both parent strains, and the concentrations of ethyl hexanoate were above the flavour threshold of $0.2 \mathrm{mg} / \mathrm{L}$ [26]. Ethyl esters give beer a fruity and apple-like aroma. The cause of these differences in ester formation of the hybrid strains compared to both parent strains is not known, but could be due to both genetic differences (e.g. increased expression and different functionality of orthologous genes [5, 37, 44]) and an indirect result of the fermentations, e.g. from the formation of more alcohol and fatty acid precursors, differences in wort $\mathrm{pH}$ or differences in yeast growth [18, 49]. The formation of acetate esters is dependent mainly on the expression and enzyme activities of the transferase-encoding ATF1 and ATF2 genes [44], while Saerens et al. [36, 37] found that the enzymes encoded by EHTI and EEBI genes are mainly responsible for ethyl ester synthesis. Overall, a low amount of esters was observed in the beer fermented with S. cerevisiae A81062. This was most likely a result of the low and sub-optimal fermentation temperature, resulting in less expression and enzyme activity of genes involved with ester synthesis, e.g. ATF1, ATF2, EEB1 and EHT1. It has been shown that the expression of these genes, encoding transferase enzymes involved in acetate and ethyl ester synthesis, is increased at increasing temperatures [37, 44]. The results suggest that it could be possible to increase aromatic diversity of lager yeast strains through interspecific hybridization and produce lager yeast strains with desired aroma production through the selection of appropriate parent strains. However, more work on the subject, especially in regard to relationships between orthologous gene expression and aroma production, must still be done. All beers also had a distinct clove-like aroma, caused by the presence of 4-vinylguaiacol [8], suggesting that the hybrid strains had inherited the $P A D 1$ gene, coding for the phenylacrylic acid decarboxylase enzyme responsible for the conversion of ferulic acid to 4-vinylguaiacol, from either of the parents, which were both $P A D 1+$ (our own unpublished data).

The flocculation assay revealed strong flocculation for both the $S$. cerevisiae A81062 parent strain and all four hybrid strains H1-H4, while S. eubayanus C12902 displayed poor flocculation (Table 2). These properties were also evident during fermentation, as more suspended biomass was observed in the fermentations with S. eubayanus than with the hybrids (Fig. 3c). The flocculation ability of yeast is defined mainly by which FLO genes are functional in various strains [47], and here the hybrid strains have clearly inherited flocculation ability from the $S$. cerevisiae parent. Surprisingly, the flocculation of the hybrid strains was stronger than both the parent strains, thus again revealing phenotype amplification (Table 2). Flocculation of yeast towards the end of fermentation allows for a cheap and effective way of removing yeast from the beer, and the use of strongly flocculating yeast strains is especially popular among smaller breweries.

In conclusion, the use of mass mating to generate de novo lager yeast hybrids through interspecific hybridization between a $S$. cerevisiae ale strain and $S$. eubayanus was successful. The resulting hybrid strains not only inherited beneficial properties from both parent strains (cryotolerance, maltotriose utilization and strong flocculation), but also showed apparent hybrid vigour compared to the parent strains by fermenting faster and achieving a more complete utilization of fermentable sugars. It must be mentioned that here, the performance of the hybrid strains was compared relative to the original parental strains and not the actual haploid spore product that mated to form the triploid hybrids. The possible heterozygosity of the parent strains suggests that spore products may be quite different genetically from any other spore product and thus also have quite different fermentation behaviour than either the parent or other sister spores [2, 41]. How natural lager yeast hybrids were first created is still not known. The clearly improved fermentation performance of the hybrid strains shown here would support the hypothesis that $S$. eubayanus may have initially been present as a contaminant in 
Acetaldehyde

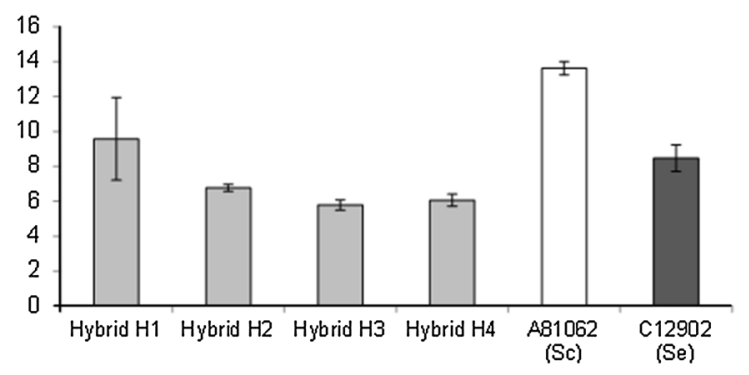

2-Methylpropanol

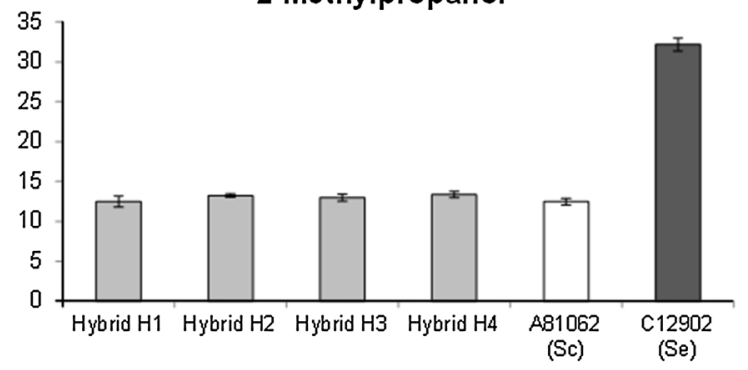

3-Methylbutanol

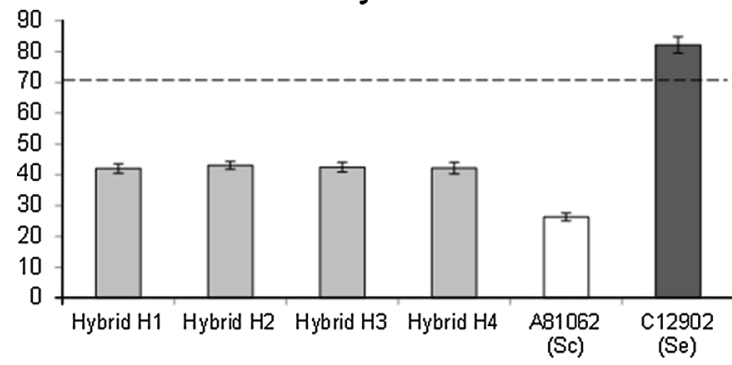

Ethyl acetate

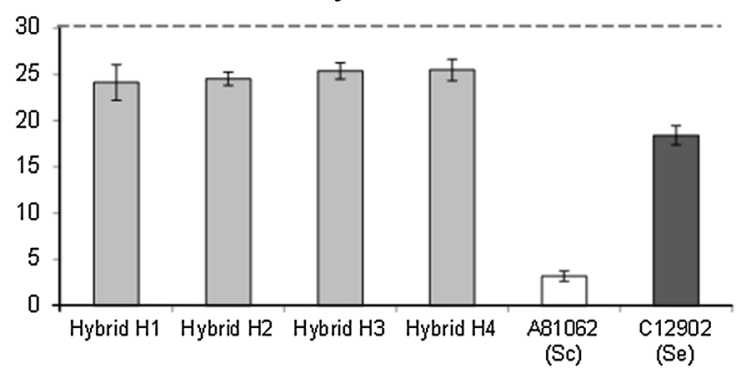

Ethyl octanoate

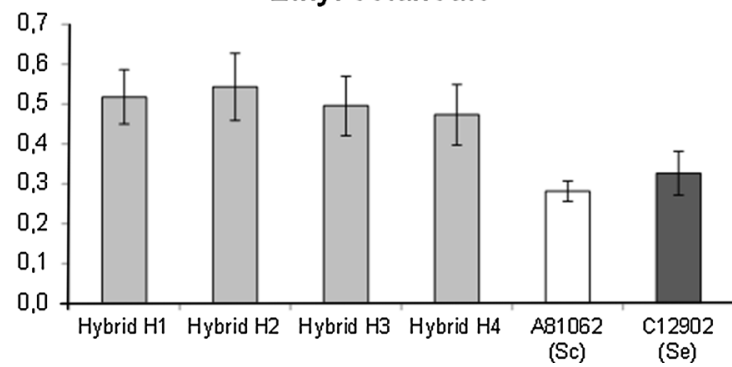

Fig. 4 The concentrations of aroma compounds in the beers fermented with the hybrid and parent strains $(\mathrm{mg} / \mathrm{L})$. Where visible, the dashed line represents the typical flavour threshold [26]. Values are
1-Propanol

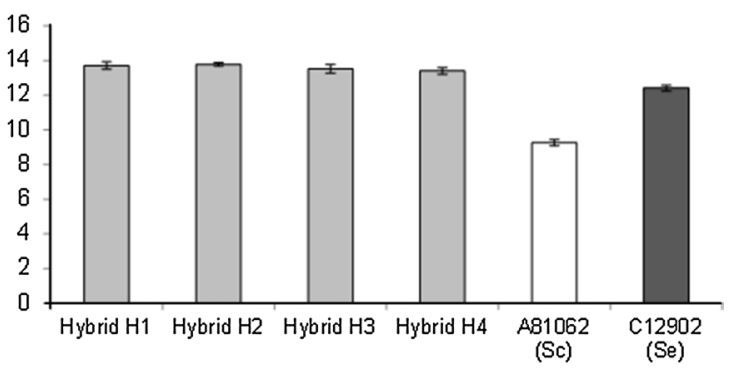

2-Methylbutanol

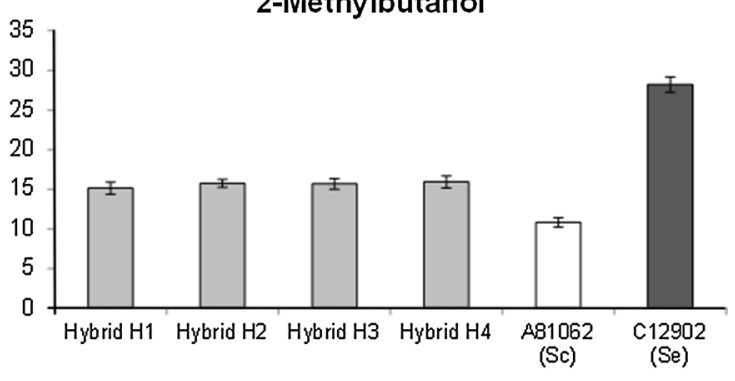

3-Methylbutyl acetate

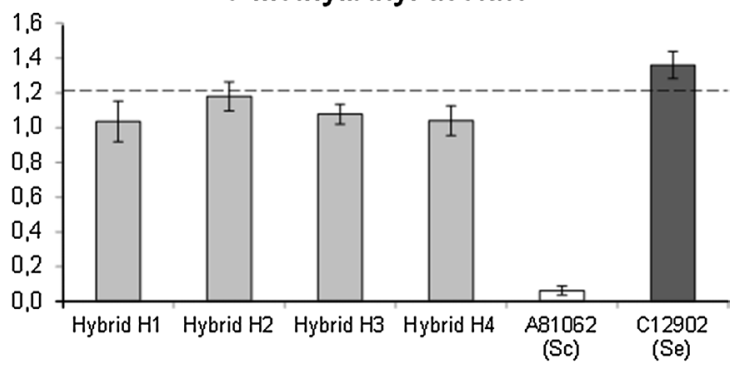

Ethyl hexanoate

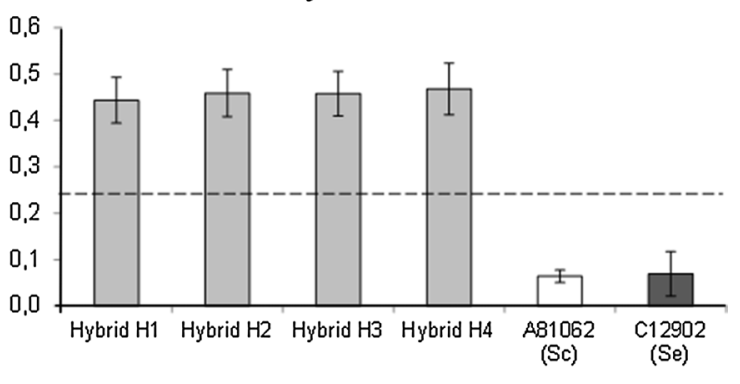

Ethyl decanoate

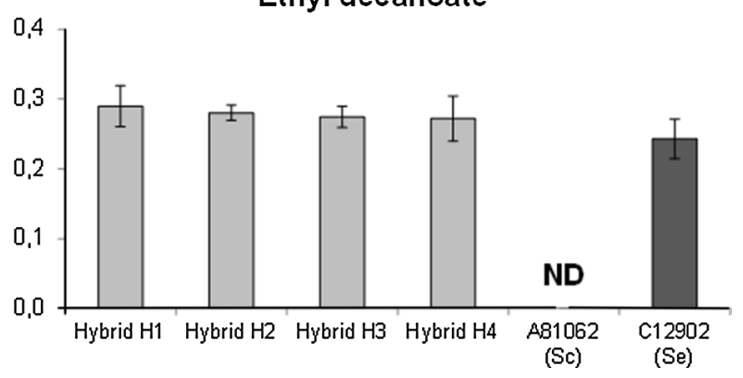

means from two independent fermentations and error bars where visible represent the range 
S. cerevisiae ale fermentations. At lower temperatures the hybrid state, which confers phenotypic benefits from both parents, would have been selected for and hybrids would have quickly dominated the brewing process $[14,42]$. The genomes of newly created yeast hybrids are also known to be unstable, and this may allow for evolutionary adaptation of hybrids to different environments [20]. These results suggest that interspecific hybridization is suitable for production of novel non-GM lager yeast strains with unique properties (e.g. flavour production or elevated stress tolerance) and generation of novel beer styles. Further investigation of the properties of de novo hybrids is required, especially in regard to the proportionality of DNA inheritance, the origin of mitochondrial DNA and aroma production and may help to elucidate the evolutionary history of industrial lager yeast strains. As a final remark, we want to mention the recent work of Marit Hebly and co-workers from Delft University of Technology entitled "Saccharomyces cerevisiae x Saccharomyces eubayanus interspecific hybrid, the best of both worlds and beyond" that further reinforces our observations and ideally complements our study. This work will be published shortly in FEMS Yeast Research.

Acknowledgments We thank Annika Wilhelmson for her support throughout, Eero Mattila and Arvi Wilpola for wort preparation and other assistance in the VTT Pilot Brewery, and Aila Siltala and Sirpa Jylhä for skilled technical assistance. This work was supported by the Alfred Kordelin Foundation, PBL Brewing Laboratory, the Academy of Finland (Academy Project 276480), and the FP7 Marie-Curie ITN YEASTCELL.

Open Access This article is distributed under the terms of the Creative Commons Attribution License which permits any use, distribution, and reproduction in any medium, provided the original author(s) and the source are credited.

\section{References}

1. Bellon J, Eglinton J, Siebert T, Pollnitz A, Rose L, de Barros Lopes M, Chambers P (2011) Newly generated interspecific wine yeast hybrids introduce flavour and aroma diversity to wines. Appl Microbiol Biotechnol 91:603-612

2. Bilinski C, Russell I, Stewart G (1986) Analysis of sporulation in brewer's yeast: induction of tetrad formation. J Inst Brew 92:594-598

3. Bisson L, Coons D, Kruckeberg A, Lewis D (1993) Yeast sugar transporters. Crit Rev Biochem Mol Biol 28:259-308

4. Boeke J, Trueheart J, Natsoulis G, Fink G (1987) 5-Fluoroorotic acid as a selective agent in yeast molecular genetics. Methods Enzymol 154:164-175

5. Bolat I, Romagnoli G, Zhu F, Pronk J, Daran J (2013) Functional analysis and transcriptional regulation of two orthologs of ARO10, encoding broad-substrate-specificity 2-oxo-acid decarboxylases, in the brewing yeast Saccharomyces pastorianus CBS1483. FEMS Yeast Res 13:505-517

6. Cebollero E, Gonzalez-Ramos D, Tabera L, Gonzalez R (2007) Transgenic wine yeast technology comes of age: is it time for transgenic wine? Biotechnol Lett 29:191-200
7. Chen Z (2010) Molecular mechanisms of polyploidy and hybrid vigor. Trends Plant Sci 15:57-71

8. Coghe S, Benoot K, Delvaux F, Vanderhaegen B, Delvaux FR (2004) Ferulic acid release and 4-vinylguaiacol formation during brewing and fermentation: indication for feruloyl esterase activity in Saccharomyces cerevisiae. J Agric Food Chem 52:602-608

9. D'Hautcourt O, Smart K (1999) Measurement of brewing yeast flocculation. J Am Soc Brew Chem 57:123-128

10. Dietvorst J, Londesborough J, Steensma HY (2005) Maltotriose utilization by lager yeast strains: MTT1 encodes a maltotriose transporter. Yeast 22:775-788

11. Dunn B, Sherlock G (2008) Reconstruction of the genome origins and evolution of the hybrid lager yeast Saccharomyces pastorianus. Genome Res 18:1610-1623

12. Garcia Sanchez R, Solodovnikova N, Wendland J (2012) Breeding of lager yeast with Saccharomyces cerevisiae improves stress resistance and fermentation performance. Yeast 29:343-355

13. Gibson B, Storgårds E, Krogerus K, Vidgren V (2013) Comparative physiology and fermentation performance of Saaz and Frohberg lager yeast strains and the parental species Saccharomyces eubayanus. Yeast 30:255-266

14. Gibson B, Liti G (2015) Saccharomyces pastorianus: genomic insights inspiring innovation for industry. Yeast 32:17-27

15. Gunge N, Nakatomi Y (1972) Genetic mechanisms of rare matings of the yeast Saccharomyces cerevisiae heterozygous for mating type. Genetics 70:41-58

16. Haase S, Reed S (2002) Improved flow cytometric analysis of the budding yeast cell cycle. Cell Cycle 1:132-136

17. Henderson C, Lozada-Contreras M, Jiranek V, Longo M, Block D (2013) Ethanol production and maximum cell growth are highly correlated with membrane lipid composition during fermentation as determined by lipidomics analysis of 22 Saccharomyces cerevisiae strains. Appl Environ Microbiol 79:91-104

18. Hiralal L, Olaniran A, Pillay B (2014) Aroma-active ester profile of ale beer produced under different fermentation and nutritional conditions. J Biosci Bioeng 117:57-64

19. Krogerus K, Gibson B (2013) Influence of valine and other amino acids on total diacetyl and 2,3-pentanedione levels during fermentation of brewer's wort. Appl Microbiol Biotechnol 97:6919-6930

20. Kunicka-Styczynska A, Rajkowska K (2011) Physiological and genetic stability of hybrids of industrial wine yeasts Saccharomyces sensu stricto complex. J Appl Microbiol 110:1538-1549

21. Libkind D, Hittinger C, Valerio E, Goncalves C, Dover J, Johnston M, Goncalves P, Sampaio J (2011) Microbe domestication and the identification of the wild genetic stock of lager-brewing yeast. Proc Natl Acad Sci USA 108:14539-14544

22. Lippman Z, Zamir D (2007) Heterosis: revisiting the magic. Trends Genet 23:60-66

23. Liti G, Peruffo A, James S et al (2005) Inferences of evolutionary relationships from a population survey of LTR-retrotransposons and telomeric-associated sequences in the Saccharomyces sensu stricto complex. Yeast 22:177-192

24. Lucero P, Herweijer M, Lagunas R (1993) Catabolite inactivation of the yeast maltose transporter is due to proteolysis. FEBS Lett 333:165-168

25. Marullo P, Bely M, Masneuf-Pomarede I, Aigle M, Dubourdieu D (2004) Inheritable nature of enological quantitative traits is demonstrated by meiotic segregation of industrial wine yeast strains. FEMS Yeast Res 4:711-719

26. Meilgaard M (1982) Prediction of flavor differences between beers from their chemical composition. J Agric Food Chem 30:1009-1017

27. Merritt A, Hurley R (1972) Evaluation of sporulation media for yeasts obtained from pathological material. J Med Microbiol $5: 21-30$ 
28. Muir A, Harrison E, Wheals A (2011) A multiplex set of speciesspecific primers for rapid identification of members of the genus Saccharomyces. FEMS Yeast Res 11:552-563

29. Paget C, Schwartz J, Delneri D (2014) Environmental systems biology of cold-tolerant phenotype in Saccharomyces species adapted to grow at different temperatures. Mol Ecol 23:5241-5257

30. Pengelly R, Wheals A (2013) Rapid identification of Saccharomyces eubayanus and its hybrids. FEMS Yeast Res 13:156-161

31. Pham T, Wimalasena T, Box W, Koivuranta K, Storgårds E, Smart K, Gibson B (2011) Evaluation of ITS PCR and RFLP for differentiation and identification of brewing yeast and brewery 'wild' yeast contaminants. J Inst Brew 117:556-568

32. Pires E, Teixeira J, Branyik T, Vicente A (2014) Yeast: the soul of beer's aroma-a review of flavour-active esters and higher alcohols produced by the brewing yeast. Appl Microbiol Biotechnol 98:1937-1949

33. Plech M, de Visser J, Korona R (2014) Heterosis is prevalent among domesticated but not wild strains of Saccharomyces cerevisiae. G3 4:315-323

34. Powell C, Casalis F, Fischborn T (2012) Stress tolerance in group 1 and group 2 lager brewing yeasts. In: Proceedings of the World Brewing Congress 2012, Portland, OR, USA July 28-August 1, 2012

35. Rainieri S, Kodama Y, Nakao Y, Pulvirenti A, Giudici P (2008) The inheritance of mtDNA in lager brewing strains. FEMS Yeast Res 8:586-596

36. Saerens D, Verstrepen K, Van Laere S, Voet A, Van Dijck P, Delvaux FR, Thevelein J (2006) The Saccharomyces cerevisiae EHT1 and EEB1 genes encode novel enzymes with mediumchain fatty acid ethyl ester synthesis and hydrolysis capacity. J Biol Chem 281:4446-4456

37. Saerens S, Verbelen P, Vanbeneden N, Thevelein J, Delvaux FR (2008) Monitoring the influence of high-gravity brewing and fermentation temperature on flavour formation by analysis of gene expression levels in brewing yeast. Appl Microbiol Biotechnol 80:1039-1051

38. Sato M, Kishimoto M, Watari J, Takashio M (2002) Breeding of brewer's yeast by hybridization between a top-fermenting yeast Saccharomyces cerevisiae and a cryophilic yeast Saccharomyces bayanus. J Biosci Bioeng 93:509-511

39. Steensels J, Meersman E, Snoek T, Saels V, Verstrepen K (2014) Large-scale selection and breeding to generate industrial yeasts with superior aroma production. Appl Environ Microbiol. doi:10.1128/AEM.02235-14
40. Stewart G, Hill A, Russell I (2013) 125th anniversary review: developments in brewing and distilling yeast strains. J Inst Brew 119:202-220

41. Tsuboi M, Takahashi T (1988) Genetic analysis of the nonsporulating phenotype of brewer's yeasts. J Ferment Technol 66:605-613

42. Walther A, Hesselbart A, Wendland J (2014) Genome sequence of Saccharomyces carlsbergensis, the world's first pure culture lager yeast. G3 4:783-793

43. van Dijken J, Bauer J, Brambilla L et al (2000) An interlaboratory comparison of physiological and genetic properties of four Saccharomyces cerevisiae strains. Enzyme Microbiol Technol 26:706-714

44. Verstrepen K, Van Laere S, Vanderhaegen B, Derdelinckx G, Dufour J, Pretorius I, Winderickx J, Thevelein J, Delvaux FR (2003) Expression levels of the yeast alcohol acetyltransferase genes ATF1, Lg-ATF1, and ATF2 control the formation of a broad range of volatile esters. Appl Environ Microbiol 69:5228-5237

45. Vidgren V, Ruohonen L, Londesborough J (2005) Characterization and functional analysis of the MAL and MPH loce for maltose utilization in some ale and lager yeast strains. Appl Environ Microbiol 71:7846-7857

46. Vidgren V, Huuskonen A, Virtanen H, Ruohonen L, Londesborough J (2009) Improved fermentation performance of a lager yeast after repair of its $A G T 1$ maltose and maltotriose transporter genes. Appl Environ Microbiol 75:2333-2345

47. Vidgren V, Londesborough J (2011) 125th anniversary review: yeast flocculation and sedimentation in brewing. J Inst Brew 117:475-487

48. Vidgren V, Londesborough J (2012) Characterization of the Saccharomyces bayanus-type AGT1 transporter of lager yeast. J Inst Brew 118:148-151

49. Yoshioka K, Hashimoto N (1981) Ester formation by alcohol acetyltransferase from brewer's yeast. Agric Biol Chem 45:2183-2190

50. Zaret K, Sherman F (1985) Alpha-aminoadipate as a primary nitrogen source for Saccharomyces cerevisiae mutants. J Bacteriol 162:579-583

51. Zheng X, D’Amore T, Russell I, Stewart G (1994) Factors influencing maltotriose utilization during brewery wort fermentations. J Am Soc Brew Chem 52:41-47

52. Storchova Z (2014) Ploidy changes and genome stability in yeast. Yeast 31:421-430 